\title{
An Analysis on Consensus Measures in Group Decision Making
}

\author{
M. J. del Moral \\ Department of \\ Statistics and \\ Operational Research \\ University of Granada \\ 18071 Granada Spain \\ Email: delmoral@ugr.es
}

\author{
F. Chiclana \\ CCI \\ Faculty of Technology \\ De Montfort University \\ Leicester LE1 9BH, UK \\ Email: chiclana@dmu.ac.uk
}

\author{
J. M. Tapia \\ Department of \\ Quantitative Methods \\ in Economic and Business \\ University of Granada \\ 18071 Granada Spain \\ Email: jmtaga@ugr.es
}

\author{
E. Herrera-Viedma \\ Department of \\ Computer Science \\ and A.I \\ University of Granada \\ 18071 Granada Spain \\ Email: viedma@decsai.ugr.es
}

\begin{abstract}
In Group Decision Making (GDM) problems before to obtain a solution a high level of consensus among experts is required. Consensus measures are usually built using similarity functions measuring how close experts' opinions or preferences are. Similarity functions are defined based on the use of a metric describing the distance between experts' opinions or preferences. Different distance functions have been proposed to implement consensus measures. This paper analyzes the effect of the application of different aggregation operators combined with the use of different distance functions for measuring consensus in GDM problems. It is concluded that the application of different aggregation operators together with different distance functions has a significant effect on the speed of achieving consensus. These results are analysed and used to derive decision support rules, based on a convergent criterion, that can be used to control the convergence speed of the consensus process using the compared distance functions.
\end{abstract}

\section{INTRODUCTION}

For reaching a decision, experts have to express their preferences by means of a set of evaluations over a set of alternatives. The known definition of consensus as as the full and unanimous agreement of all the experts regarding all the feasible alternatives may become inconvenient because it only allows differentiating between two states, namely, the existence and absence of consensus. Another significance of the concept of consensus refers to the judgement arrived at by 'most of' those concerned, which has led to the definition and use of a new concept of consensus degree referred to as 'soft' consensus degree [2], [13], [16].

Based on the use of such soft consensus measure, the consensus process can be modelled as a dynamic and iterative group discussion process, coordinated by a moderator, who helps the experts to make their opinions closer.

Soft consensus measures represent the level of agreement among experts, and therefore their definition is based on the concept of similarity between their opinions (preferences). The evaluation of consensus necessarily implies the computation and aggregation of the 'distance' representing disagreement between the opinions (preferences) of each pair of experts on each pair of alternatives [3], [19]. An issue here is that the convergence of the consensus process towards a solution acceptable by most of the experts could be affected by the particular distance function and the aggregation operator used to measure disagreement [1], [4], [20], [21], [24].

In Chiclana et al. [9], by using nonparametric Wilcoxon tests, significant differences were found between the behaviour of five of the most commonly used distance functions in modelling soft consensus measures, Manhattan, Euclidean, Cosine, Dice, and Jaccard, and their behaviour were further analysed using a convergent criterion and a set of rules were identified for their application to control the speed of convergence towards consensus. The aim of this paper is to analyze the effect of the application of several aggregation operators (Maximum, Minimum and Average) together with the use of different distance functions in GDM.

This paper is structured as follows. Section II introduces concepts essential to the understanding of the rest of the paper: the GDM problem (Subsection II-A), the selection process (Subsection II-B) and the consensus process (Subsection II-C). Section III describes the design of the experiment and the results obtained in evaluating the different distance functions for measuring consensus in GDM problems. Finally, Section IV concludes the paper.

\section{PRELIMINARIES}

In this section, we will introduce the basic notions of GDM problem and Consensus Model.

\section{A. The GDM Problem}

GDM problems consist in finding the best alternative(s) from a set of feasible alternatives $X=\left\{x_{1}, \ldots, x_{n}\right\}$ according to the preferences provided by a group of experts $E=$ $\left\{e_{1}, \ldots, e_{m}\right\}$. Different preference elicitation methods were compared in [17], where it was concluded that pairwise comparison methods are more accurate than non-pairwise methods [23].

Definition 1 (Fuzzy Preference Relation): A fuzzy preference relation $P$ on a finite set of alternatives $X$ is characterized by a membership function $\mu_{P}: X \times X \longrightarrow[0,1], \mu\left(x_{i}, x_{j}\right)=$ $p_{i j}$, verifying

$$
p_{i j}+p_{j i}=1 \forall i, j \in\{1, \ldots, n\} .
$$


When cardinality of $X$ is small, the fuzzy preference relation may be conveniently denoted by the matrix $P=\left(p_{i j}\right)$. The following interpretation is also usually assumed:

- $p_{i j}=1$ indicates the maximum degree of preference for $x_{i}$ over $x_{j}$.

- $\left.p_{i j} \in\right] 0.5,1\left[\right.$ indicates a definite preference for $x_{i}$ over $x_{j}$.

- $p_{i j}=0.5$ indicates indifference between $x_{i}$ and $x_{j}$.

Two different processes are applied in GDM problems before a final solution can be obtained [5], [14], [18]: (1) the consensus process and (2) the selection process. The consensus process refers to how to obtain the maximum degree of consensus or agreement between the set of experts. The selection process obtains the final solution according to the preferences [6] given by the experts.

\section{B. Selection Process}

The selection process involves two different steps [12]: (i) aggregation of individual preferences and (ii) exploitation of the collective preference.

a) Aggregation phase: This phase defines a collective preference relation, $P^{c}=\left(p_{i j}^{c}\right)$, obtained by means of the aggregation of all individual fuzzy preference relations $\left\{P^{1}, P^{2}, \ldots, P^{m}\right\}$, and indicates the global preference between every pair of alternatives according to the majority of experts' opinions.

The aggregation operation by means of a quantifier guided OWA operator, $\phi_{Q}$, is carried out as follows:

$$
p_{i j}^{c}=\phi_{Q}\left(p_{i j}^{1}, \ldots, p_{i j}^{m}\right)=\sum_{k=1}^{m} w_{k} \cdot p_{i j}^{\sigma(k)}
$$

where $\sigma$ is a permutation function such that $p_{i j}^{\sigma(k)} \geq$ $p_{i j}^{\sigma(k+1)}, \forall k=1, \ldots, \mathrm{m}-1 ; Q$ is a fuzzy linguistic quantifier [26] of fuzzy majority and it is used to calculate the weighting vector of $\phi_{Q}, W=\left(w_{1}, \ldots, w_{n}\right)$ such that, $w_{k} \in[0,1]$ and $\sum_{k=1}^{n} w_{k}=1$, according to the following expression [25]:

$$
w_{k}=Q(k / n)-Q((k-1) / n), \forall k \in\{1, \ldots, n\} .
$$

$Q$ being a linguistic quantifier representing the concept of fuzzy majority by means of the following expression

$$
Q(r)= \begin{cases}0 & \text { if } 0 \leq r<a \\ \frac{r-a}{b-a} & \text { if } a \leq r \leq b \\ 1 & \text { if } b<r \leq 1\end{cases}
$$

Alternative representations for the concept of fuzzy majority can be found in the literature [7]. In this study we use three different OWA operators, i.e., Maximum $(W=$ $[1,0, \ldots, 0])$, Minimum $(W=[0, \ldots, 0,1])$, and Average $(W=[1 / n, 1 / n, \ldots, 1 / n])$.

b) Exploitation phase: This phase transforms the global information about the alternatives into a global ranking of them, from which the set of solution alternatives is obtained.

Clearly, it is preferable that the experts achieve a high level of consensus concerning their preferences before applying the selection process.

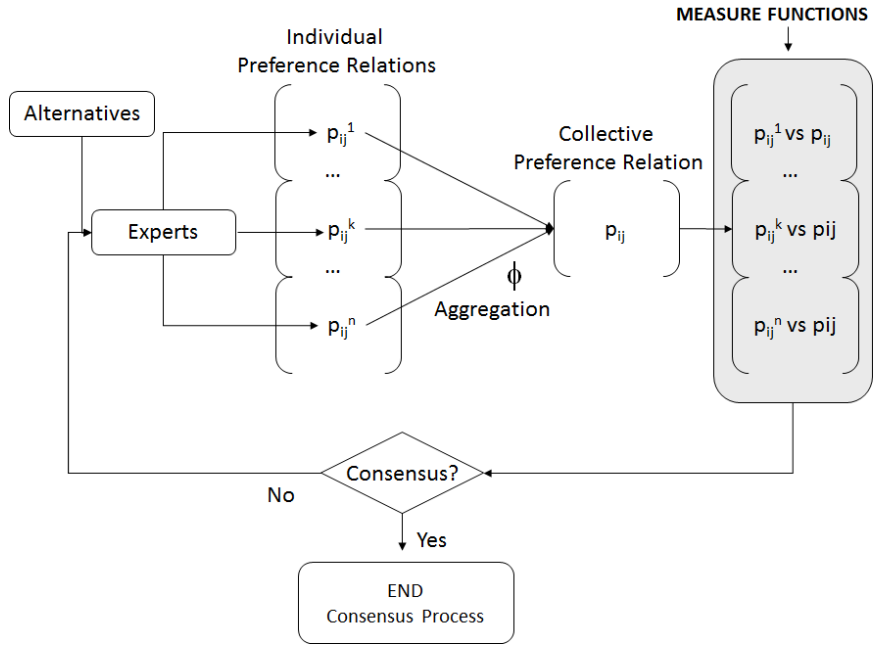

Fig. 1. Applying Measure Functions

\section{Consensus Model}

The computation of the level of agreement among experts involves necessarily the measurement of the distance or, equivalently, the similarity between their preference values. In the following, we provide the formal definition of distance and similarity functions as given in [11]:

Definition 2 (Distance): Let $A$ be a set. A function $d: A \times$ $A \longrightarrow \mathbb{R}$ is called a distance (or disimilarity) on $A$ if, for all $x, y \in A$, there holds

1) $d(x, y) \geq 0$ (non-negativity)

2) $d(x, y)=d(y, x)$ (symmetry)

3) $d(x, x)=0$ (reflexivity)

Definition 3 (Similarity): Let $A$ be a set. A function $s: A \times$ $A \longrightarrow \mathbb{R}$ is called a similarity on $A$ if $s$ is non-negative, symmetric, and if $s(x, y) \leq s(x, x)$ holds for all $x, y \in A$, with equality if and only if $x=y$.

The main transformations between a distance $d$ and a similarity $s$ bounded by 1 are [11]:

$$
\begin{array}{r}
d=1-s ; d=\frac{1-s}{s} ; \quad d=\sqrt{1-s} \\
d=\sqrt{2\left(1-s^{2}\right)} ; \quad d=\arccos s \\
d=-\ln s
\end{array}
$$

The similarity function is used for measuring both consensus degrees and proximity measures. The first ones are calculated by fusing the similarity of the preference values of all the experts on each pair of alternatives as per the expression (6) below. The second ones are calculated by measuring the similarity between the preferences of each expert in the group and the collective preferences, previously obtained by fusing all the individual experts' preferences.

The main problem is how to find a way of making individual positions converge and, therefore, how to support the experts in obtaining and agreeing with a particular solution. To do this, a consensus level required for that solution is fixed 
in advance. This consensus model has been widely investigated in [14] and [8].

The computation of consensus degrees is carried out as follows:

1) The proximity between the preference values provided by each expert, $r$, and the corresponding preference values of the rest of the experts in the group is measured and recorded in a similarity matrix, $S M^{r}=\left(s m_{i j}^{r}\right)$, with

$$
s m_{i j}^{r}=s\left(\mathbf{p}_{\mathbf{i j}}^{\mathbf{r}}, \mathbf{p}_{\mathbf{i j}}\right)
$$

where:

$$
\begin{aligned}
& \mathbf{p}_{\mathbf{i j}}^{\mathbf{r}}=\left(p_{i j}^{r}, \ldots, p_{i j}^{r}\right), \\
& \mathbf{p}_{\mathbf{i j}}=\left(p_{i j}^{1}, \ldots, p_{i j}^{r-1}, p_{i j}^{r+1}, \ldots, p_{i j}^{m}\right) \text { and } \\
& s:[0,1]^{m-1} \times[0,1]^{m-1} \rightarrow[0,1] \text { is a similarity function. }
\end{aligned}
$$

The closer $s m_{i j}^{r}$ to 1 the more similar $\mathbf{p}_{\mathbf{i j}}^{\mathbf{r}}$ and $\mathbf{p}_{\mathbf{i j}}$ are, while the closer $s m_{i j}^{r}$ to 0 the more distant $\mathbf{p}_{\mathbf{i j}}^{\mathbf{r}}$ and $\mathbf{p}_{\mathbf{i j}}$ are.

2) A consensus matrix, $C M=\left(\mathrm{cm}_{i j}\right)$, is obtained by aggregating, using an OWA operator $(\phi)$, all the similarity matrices obtained via Equation (6):

$$
\forall i, j \in\{1, \ldots, n\}: c m_{i j}=\phi\left(s m_{i j}^{1}, \ldots, s m_{i j}^{m}\right)
$$

3) Consensus degrees are defined in each one of the three different levels of a fuzzy preference relation:

Level 1. Consensus on the pairs of alternatives, $c p_{i j}$. It measures the agreement among all experts on the pair of alternatives $\left(x_{i}, x_{j}\right)$ :

$$
\forall i, j=1, \ldots, n \wedge i \neq j: c p_{i j}=c m_{i j}
$$

Level 2. Consensus on alternatives, $c a_{i}$. It measures the agreement among all experts on the alternative $x_{i}$, and it is obtained by aggregating the consensus degrees of all the pairs of alternatives involving it:

$$
c a_{i}=\phi\left(c p_{i j}, c p_{j i} ; j=1, \ldots, n \wedge j \neq i\right)
$$

Level 3. Consensus on the relation, $c r$. It measures the global agreement among all experts, and it is obtained by aggregating all the consensus degrees at the level of pairs of alternatives:

$$
c r=\phi\left(c a_{i} ; i=1, \ldots, n\right)
$$

\section{COMPARATIVE STUdy: EXPERIMENTAL DESIGN AND RESULT}

Given two vectors of real numbers $\mathbf{a}=\left(a_{1}, \ldots, a_{n}\right)$ and $\mathbf{b}=\left(b_{1}, \ldots, b_{n}\right)$, the following five distance functions have been considered in our study [11]:

$$
\begin{array}{r}
d_{1}(\mathbf{a}, \mathbf{b})=\sum_{i=1}^{n}\left|a_{i}-b_{i}\right| \\
d_{2}(\mathbf{a}, \mathbf{b})=\sqrt{\sum_{i=1}^{n}\left|a_{i}-b_{i}\right|^{2}}
\end{array}
$$

$$
\begin{gathered}
d_{3}(\mathbf{a}, \mathbf{b})=\frac{\sum_{i=1}^{n} a_{i} \cdot b_{i}}{\sqrt{\sum_{i=1}^{n} a_{i}^{2} \cdot \sqrt{\sum_{i=1}^{n} b_{i}^{2}}}} \\
d_{4}(\mathbf{a}, \mathbf{b})=\frac{2 \cdot \sum_{i=1}^{n} a_{i} \cdot b_{i}}{\sum_{i=1}^{n} a_{i}^{2}+\sum_{i=1}^{n} b_{i}^{2}} \\
d_{5}(\mathbf{a}, \mathbf{b})=\frac{\sum_{i=1}^{n} a_{i} \cdot b_{i}}{\sum_{i=1}^{n} a_{i}^{2}+\sum_{i=1}^{n} b_{i}^{2}-\sum_{i=1}^{n} a_{i} \cdot b_{i}}
\end{gathered}
$$

where $d_{1}$ denotes Manhattan distance function, $d_{2}$ Euclidean distance function, $d_{3}$ Cosine distance function, $d_{4}$ Dice distance function and $d_{5}$ Jaccard distance function.

We assumed the alternative hypothesis [10]:

The application of the Manhattan, Euclidean, Cosine, Dice and Jaccard distance functions in GDM problems produce significant differences in the measurement of consensus

tested and corroborated in [9].

A total of twelve (12) random GDM problems were generated for each one of the possible combinations of experts $(m=4,6,8,10)$ and alternatives $(n=4,6,8)$. Each one of these random GDM problems was executed three (3) times, each time using one of the three different OWA operators given in Subsection II-B to compute the consensus degrees at the relation levels.

Table I shows the level of consensus in percentage achieved by the different distance functions for each GDM problem, showing only the number of experts as the variable parameter, and for each one of our aggregation operators. The greater a value in this table the greater the global level of consensus achieved by the experts in the corresponding GDM problem. The comparison of column entries could be used to find out which distance function returns the largest values and therefore it could lead to a faster convergence of the consensus process.

From Table I we can conclude the following:

1) The Manhattan $\left(d_{1}\right)$ and the Euclidean $\left(d_{2}\right)$ distance functions increase the global consensus level as the number of experts increases. Also, the values of consensus returned by both distance functions are quite similar, which was already corroborated by the results obtained in [9].

2) The Cosine $\left(d_{3}\right)$ and Dice $\left(d_{4}\right)$ distance functions result in fairly similar and stable global consensus levels regardless of the number of experts. For low number of experts both tend to produce higher values of consensus than the Manhattan $\left(d_{1}\right)$ and the Euclidean $\left(d_{2}\right)$ distance functions, which reverse when the number of experts is 8 or higher for "Average" and "Maximum" OWA operator. 
TABLE I. CONSENSUS DEgREE IN PERCENTAGES For ALL GDM PROBLEMS AT THE LEVEL OF THE RELATION

\begin{tabular}{|c|c|c|c|c|}
\hline \multicolumn{5}{|c|}{ "Average" OWA } \\
\hline$d_{i} \backslash \mathrm{E}$ & 4 & 6 & 8 & 10 \\
\hline$d_{1}$ & 70,18 & 87,45 & 95,43 & 100,0 \\
\hline$d_{2}$ & 69,99 & 87,33 & 95,33 & 99,94 \\
\hline$d_{3}$ & 88,23 & 89,03 & 88,89 & 88,14 \\
\hline$d_{4}$ & 88,15 & 88,93 & 88,79 & 88,04 \\
\hline$d_{5}$ & 71,47 & 72,41 & 72,22 & 71,28 \\
\hline \multicolumn{5}{|c|}{ "Maximum" OWA } \\
\hline$d_{i} \backslash \mathrm{E}$ & 4 & 6 & 8 & 10 \\
\hline$d_{1}$ & 55,43 & 73,92 & 82,40 & 88,46 \\
\hline$d_{2}$ & 57,37 & 75,18 & 84,11 & 89,54 \\
\hline$d_{3}$ & 94,84 & 98,22 & 98,89 & 100,0 \\
\hline$d_{4}$ & 94,75 & 98,17 & 98,80 & 99,95 \\
\hline$d_{5}$ & 82,75 & 87,91 & 88,91 & 90,81 \\
\hline \multicolumn{5}{|c|}{ "Minimum" OWA } \\
\hline$d_{i} \backslash \mathrm{E}$ & 4 & 6 & 8 & 10 \\
\hline$d_{1}$ & 77,32 & 90,85 & 96,68 & 100,0 \\
\hline$d_{2}$ & 75,69 & 90,32 & 95,79 & 99,47 \\
\hline$d_{3}$ & 71,93 & 68,67 & 66,16 & 63,32 \\
\hline$d_{4}$ & 71,89 & 68,58 & 66,09 & 63,27 \\
\hline$d_{5}$ & 53,81 & 50,20 & 47,56 & 44,75 \\
\hline
\end{tabular}

Nevertheless the Manhattan $\left(d_{1}\right)$ and the Euclidean $\left(d_{2}\right)$ distance functions are always higher for "Minimum" OWA operator.

3) The Jaccard distance function $\left(d_{5}\right)$ produces the lowest global consensus levels, being fairly stable in value regardless of the number of experts but not as much as the Cosine $\left(d_{3}\right)$ and Dice $\left(d_{4}\right)$ distance functions.

4) For "Minimum" OWA operator the Manhattan $\left(d_{1}\right)$ and the Euclidean $\left(d_{2}\right)$ distance functions increase as the number of expert increases meanwhile the Cosine $\left(d_{3}\right)$ and Dice $\left(d_{4}\right)$ distance functions decrease.

\section{A. Consensus Process Convergence Rules}

Based on the above analysis we can draw rules to speed up or slow down the convergence of the consensus that could prove an important decision support tool in GDM problems.

- The Manhattan $\left(d_{1}\right)$ and the Euclidean $\left(d_{2}\right)$ distance functions help the consensus process to converge faster than the rest as they consistently produce the highest consensus values for the most of possible combinations of the number of experts and aggregation operators, particularly for "Minimum" OWA operator, except for "Maximum" OWA operator

- The Cosine $\left(d_{3}\right)$ and Dice $\left(d_{4}\right)$ distance functions are placed in a mid term position in terms of helping speed up the convergence of the consensus process, except for "Maximum" OWA operator. (See Fig. ?? a)).

- The Jaccard distance function $\left(d_{5}\right)$ contributes the least to the speed of convergence of the consensus process except for "Maximum" OWA operator.

- The Manhattan $\left(d_{1}\right)$ and the Euclidean $\left(d_{2}\right)$ distance functions are quite sensitive to the number of experts, i.e. they produce significant different consensus values when the number of expert changes.

- The Cosine $\left(d_{3}\right)$, the Dice $\left(d_{4}\right)$ and the Jaccard $\left(d_{5}\right)$ distance functions are quite stable in the global consensus values they produce with respect to changes in the number of experts.

To corroborate the above rules, we run a GDM problem with different number of experts using the three aggregation operators with the five different distance functions and record the number of rounds necessary for the consensus process to reach the threshold consensus level acceptable for the GDM to reach a solution of consensus. This is graphically represented in Fig. 2, Fig. 3 and Fig. 4.

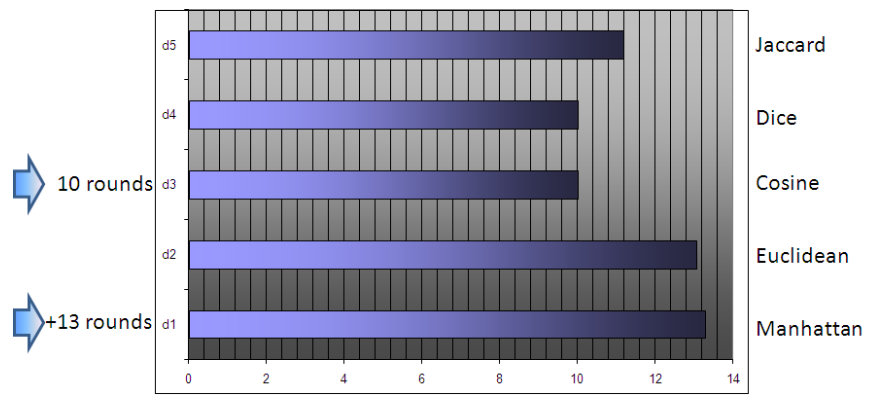

Fig. 2. Number of consensus rounds necessary for each distance function to reach consensus threshold in a GDM problem: 6 experts and "Maximum" operator

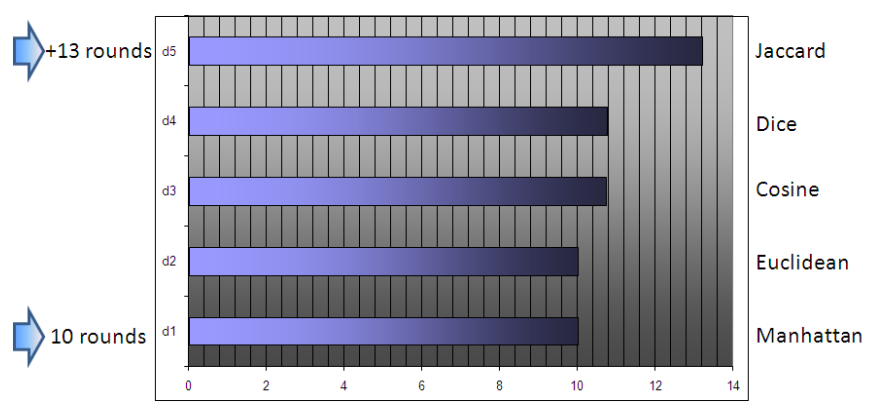

Fig. 3. Number of consensus rounds necessary for each distance function to reach consensus threshold in a GDM problem: 8 experts and "Average" operator

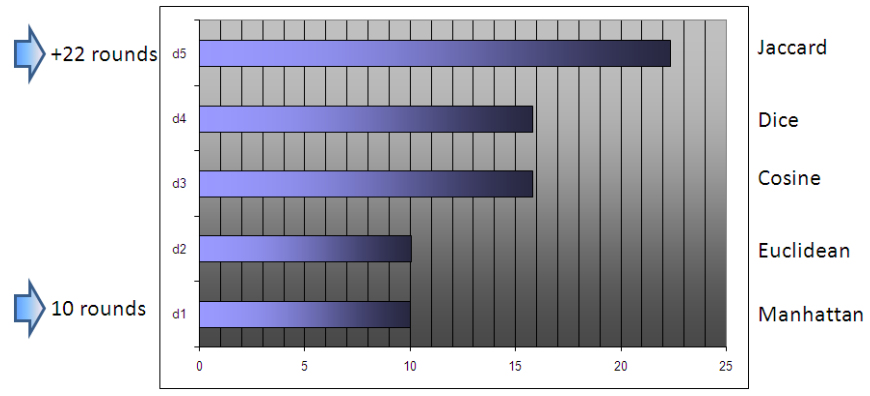

Fig. 4. Number of consensus rounds necessary for each distance function to reach consensus threshold in a GDM problem: 10 experts and "Minimum" operator

\section{CONCLUSION}

In this paper we have analyzed different distance functions and different aggregation operators used to compute consensus measures. The results in our experimental study has shown that the compared distance functions and aggregation operators 
produce significant different results in most of the GDM problems carried out. The analysis of the results allows to draw a set of rules for the application of the compared distance functions and aggregation operators that can be used to control the convergence speed of the consensus process using the compared distance functions.

\section{ACKNOWLEDGMENT}

The authors would like to acknowledge FEDER financial support from the project TIN2013-40658-P and the Andalusian Excellence Project TIC-05991 and also the research project MTM2012-35591.

\section{REFERENCES}

[1] S. Alonso, I.J. Pérez, F.J. Cabrerizo, E. Herrera-Viedma, A Linguistic Consensus Model for Web 2.0 Communities, Applied Soft Computing 13(1):149-157, 2013.

[2] F.J. Cabrerizo, F. Chiclana, R. Al-Hmouz, A. Morfeq, A.S. Balamash, E. Herrera-Viedma, Fuzzy decision making and consensus: challenges, Journal of Intelligent \& Fuzzy Systems 29:3 1109-1118, 2015.

[3] F.J. Cabrerizo, J.M. Moreno, I.J. Pérez, E. Herrera-Viedma, Analyzing consensus approaches in fuzzy group decision making: advantages and drawbacks, Soft Computing 14(5):451-463, 2010

[4] F.J. Cabrerizo, J.A. Morente-Molinera, I.J. Pérez, J. López-Gijón, E. Herrera-Viedma, A decision support system to develop a quality management in academic digital libraries, Information Sciences 323:48-58, 2015

[5] F.J. Cabrerizo, M.R. Ureña, W. Pedrycz, E. Herrera-Viedma, Building consensus in group decision making with an allocation of information granularity, Fuzzy Sets and Systems 255:115-127, 2014.

[6] F. Chiclana, F. Herrera, E. Herrera-Viedma, Integrating three representation models in fuzzy multipurpose decision making based on fuzzy preference relations, Fuzzy Sets and Systems 97(1):33-48, 1998.

[7] F. Chiclana, E. Herrera-Viedma, F. Herrera, S. Alonso, Some induced ordered weighted averaging operators and their use for solving group decision-making problems based on fuzzy preference relations, European Journal of Operational Research 182(1):383-399, 2007.

[8] F. Chiclana, F. Mata, L. Martinez, E. Herrera-Viedma, S. Alonso, Integration of a consistency control module within a consensus model, International Journal of Uncertainty, Fuzziness and Knowledge-Based Systems 16 (Supplement 1):35-53, 2008.

[9] F. Chiclana, J.M. Tapia García, M.J. Del Moral Ávila, E. HerreraViedma, A statistical comparative study of different similarity measures of consensus in GDM, Information Sciences 221:110-123, 2013.

[10] M.J. Del Moral Ávila, J.M. Tapia García, Técnicas estadísticas aplicadas, Grupo Editorial Universitario, Granada, 2006.

[11] M.M. Deza, E. Deza, Encyclopedia of Distances, Springer, Berlin, Heidelberg, 2009.

[12] F. Herrera, E. Herrera-Viedma, J.L. Verdegay, Choice processes for nonhomogeneous group decision making in linguistic setting, Fuzzy Sets and Systems 94(3):287-308, 1998.

[13] E. Herrera-Viedma, F.J. Cabrerizo, J. Kacprzyk, W. Pedrycz, A Review of Soft Consensus Models in a Fuzzy Environment, Information Fusion 17: 4-13, 2014.

[14] E. Herrera-Viedma, F. Herrera, F. Chiclana, A consensus model for multiperson decision making with different preference structures, IEEE Transactions on Systems, Man and Cybernetics, Part A: Systems and Humans 32(3):394-402, 2002.

[15] J. Kacprzyk, Group decision making with a fuzzy linguistic majority, Fuzzy Sets and Systems 18(2):105-118, 1986.

[16] J. Kacprzyk, M. Fedrizzi, Soft consensus measures for monitoring real consensus reaching processes under fuzzy preferences, Control and Cybernetics 15:309-323, 1986.

[17] I. Millet, The effectiveness of alternative preference elicitation methods in the analytic hierarchy process, Journal of Multi-Criteria Decision Analysis 6(1):41-51, 1997.
[18] J.A Morente-Molinera, R. Wikström, E. Herrera-Viedma, C. Carlsson, A linguistic mobile decision support system based on fuzzy ontology to facilitate knowledge mobilization, Decision Support Systems 81:66-75, 2016.

[19] I.J. Pérez, F.J. Cabrerizo, S. Alonso, E. Herrera-Viedma, A New Consensus Model for Group Decision Making Problems with Non Homogeneous Experts, IEEE Transactions on Systems, Man, and Cybernetics: Systems, 44(4):494-498, 2014.

[20] I.J. Pérez, F.J. Cabrerizo, E. Herrera-Viedma, A Mobile Decision Support System for Dynamic Group Decision Making Problems, IEEE Transactions on Systems, Man and Cybernetics - Part A: Systems and Humans, 40(6):1244-1256, 2010.

[21] J.M. Tapia García, M.J. del Moral, M.A. Martinez, E. Herrera-Viedma, A Consensus Model For Group Decision Making Problems With Linguistic Interval Fuzzy Preference Relations, Expert System With Applications 39(11):10022-10030, 2012.

[22] G. Salton, M.J. McGill, Introduction to modern information retrieval, Mc-Graw Hill, New York, 1983.

[23] R. Ureña, F. Chiclana, J.A Morente-Molinera, E. Herrera-Viedma, Managing Incomplete Preference Relations in Decision Making: A Review and Future Trends, Information Sciences 302(1):14-32, 2015.

[24] J. Wu, F. Chiclana, E. Herrera-Viedma, Trust Based Consensus Model for Social Network in an Incomplete Linguistic Information Context, Applied Soft Computing 35:827-839, 2015.

[25] R.R. Yager, On ordered weighted averaging aggregation operators in multicriteria decisionmaking, IEEE Transactions on Systems, Man and Cybernetics, 18(1):183-190, 1988.

[26] L.A. Zadeh, A computational approach to fuzzy quantifiers in natural languages, Computers \& Mathematics with Applications 9(1):149-184, 1983. 